\title{
Manipulating the exciton fine structure of single CdTe/ZnTe quantum dots by an in-plane magnetic field
}

\author{
K. Kowalik, ${ }^{1,2}$, 田 O. Krebs, ${ }^{2}$ A. Golnik, ${ }^{1}$ J. Suffczyński, ${ }^{1}$ P. Wojnar, ${ }^{3}$ J. Kossut, ${ }^{3}$ J. A. Gaj, ${ }^{1}$ and P. Voisin ${ }^{2}$ \\ ${ }^{1}$ Institute of Experimental Physics, Warsaw University, Hoża 69, 00-681 Warszawa, Poland \\ ${ }^{2}$ Laboratoire de Photonique et Nanostructures-CNRS, Route de Nozay, 91460 Marcoussis, France \\ ${ }^{3}$ Institute of Physics, Polish Academy of Sciences, Al. Lotników 32/46, 02-668 Warszawa, Poland
}

(Dated: 17th April 2018)

\begin{abstract}
Polarization resolved photoluminescence (PL) spectroscopy of individual CdTe/ZnTe quantum dots is investigated in the presence of external in-plane magnetic field. We find that the excitonic fine structure strongly depends on the magnitude and direction of applied field. The splitting between "bright" and "dark" states increases with the magnetic field, whereas the anisotropic exchange splitting of the bright excitons can be reduced or enhanced, depending on the field direction. Increase (decrease) is observed when the field is applied parallel to the PL polarization direction of the lower (upper) energy exciton. For intermediate fields, we observe a rotation of the PL polarization orientation. The results are discussed in terms of an effective spin Hamiltonian derived for the exciton ground state.
\end{abstract}

PACS numbers: 71.35.Ji,71.70.Gm,73.21.La,75.75.+a

\section{INTRODUCTION}

Semiconductor quantum dots (QDs) are unique non-classical light emitters. In addition to their now well-known single photon emission properties, their potential as sources of entangled photons on demand ${ }^{1,2.3}$ was recently demonstrated. The main obstacle to polarization entanglement of photons emitted in the biexciton-exciton radiative cascade is the lifted of degeneracy of the two optically active exciton states due to the anisotropic electron-hole exchange interaction. It is revealed in experiment by a splitting of the exciton and biexciton lines into doublets with orthogonal linear polarizations. The origin of the symmetry breakdown governing this fine structure splitting (FSS) is not fully established. FSS can result from any combination of in-plane shape anisotropy of a dot (elongation of the dot due to preferential growth direction), piezoelectric potential in the dot vicinity (due to the vertically asymmetric strain field), and local symmetry breakdown due to chemical bond alignment at the dot interfaces ${ }^{4.5}$. As controlling FSS is of utmost importance for quantum optics applications, different strategies for restoring higher symmetry were tested, either by influencing material properties of heterostructures (annealing or strain engineering6.7) or by applying external perturbations compensating the native asymmetry : in-plane electric field ${ }^{8}$, uniaxial strain ${ }^{9}$, and in-plane magnetic field ${ }^{2.10}$ were tried. The last-mentioned method has given the most satisfactory results so far in GaAs-based selfassembled QDs. II-VI systems have promising features in this context, based on their more robust excitonic states allowing to study non-classical light emission at higher temperatures 11 . However, they generally exhibit stronger anisotropy splittings $\frac{12}{2}$, so it is essential to devise efficient methods of symmetry control suited to II-VI QDs. In this paper we report a study of exciton
FSS in $\mathrm{CdTe} / \mathrm{ZnTe}$ QDs in the presence of an in-plane magnetic field and show that it can be increased or decreased, depending on the in-plane field direction.

\section{SAMPLE AND EXPERIMENT}

The studied sample was grown by MBE on a (001)oriented GaAs substrate. It consists of following layers: a thick $(4.3 \mu \mathrm{m})$ CdTe buffer, followed by a $0.35 \mu \mathrm{m}$ ZnTe barrier, a CdTe quantum dot layer, and a $114 \mathrm{~nm}$ ZnTe cap. QD formation was induced by desorption of amorphous Tellurium deposited on six monolayers of $\mathrm{CdTe}^{13}$. Before the cap deposition the dot layer was annealed in-situ at $480^{\circ} \mathrm{C}$ for 25 minutes. The sample emission is characterized by a broad micro-luminescence $(\mu \mathrm{PL})$ spectrum, with well resolved individual QD lines appearing on the low energy tail. For the experiment the sample was mounted directly on a specially designed Cassegrain-type microscope objective immersed in liquid helium ${ }^{14}$, in a cryostat with superconducting coil allowing to apply magnetic field up to $7 \mathrm{~T}$. The field was applied parallel to one of the $\langle 110\rangle$ crystallographic axes, in Voigt configuration. For the excitation, a CW doubled YAG laser at $532 \mathrm{~nm}$ was focused on a $1-2 \mu m^{2}$ area spot on the sample surface. The photoluminescence signal was collected by the same objective, analyzed using a linear polarizer, filtered by a monochromator, and recorded by a CCD camera. Excellent mechanical stability of this set-up enabled PL measurement of a single QD for many hours. For well isolated dots, lineshape fits allowed us to determine the line position with a precision of $30 \mu \mathrm{eV}$. A distinctive characteristic of CdTe quantum dots is that their optical axes are randomly oriented $^{15.16}$, contrarily to InAs QDs where they are clamped to the $\langle 110\rangle$ direction. This allows simultaneous measurements of different relative orientations of dot 
and applied field in a fixed geometry, by selecting different dots and rotating the analyzer accordingly. We define the dot orientation as the polarization direction of the lower component of the excitonic doublet and denote $\theta$ the angle between the field and this direction.

\section{RESULTS}
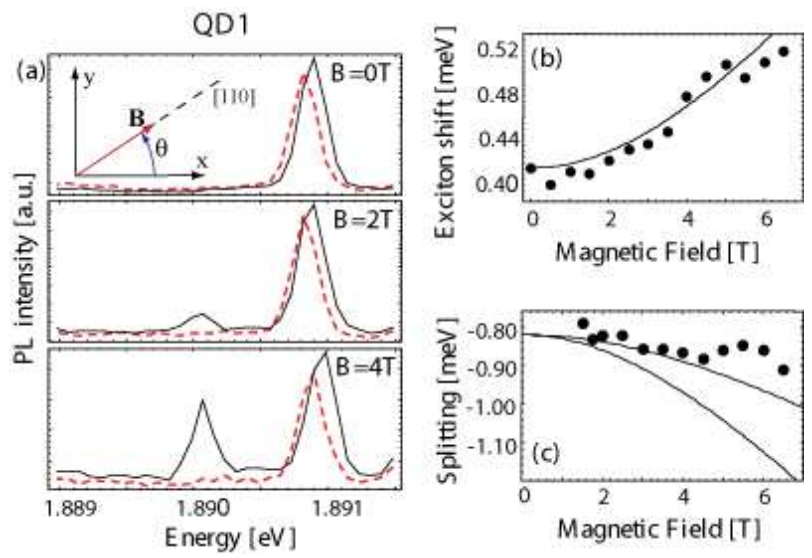

Figure 1: (Color online)(a) Linearly polarized excitonic doublet (solid and dashed lines) for magnetic fields $B=0,2$, and $4 \mathrm{~T}$ in a dot characterized by $\theta=20^{\circ}$. Inset shows the direction of the magnetic field with respect to the QD polarization eigenaxes.(b) Mean energy of the "bright" excitonic doublet versus magnetic field and (c) splitting of bright to dark states vs magnetic field. For (b) and (c) Lines are theoretical curves calculated with the model described in the text.

The polarization-resolved $\mu$-PL spectra of a QD at various fields are shown in Fig. 1 1 for the peculiar case of a dot nearly oriented along $\boldsymbol{B}\left(\theta=20^{\circ}\right)$. The most salient feature is a line appearing $\sim 1 \mathrm{meV}$ below the excitonic doublet, and developing when the field is increased. This line is attributed to the dark exciton that becomes optically active due to field-induced mixing of bright and dark states 17 . Detailed analysis shows in general a blueshift of the excitonic doublet $(105 \mu \mathrm{eV}$ at $7 \mathrm{~T})$, a similar increase of the bright-dark exciton splitting, and in this case, an increase of FSS from $87 \mu \mathrm{eV}$ at $B=0$ to $185 \mu \mathrm{eV}$ at $B=7 \mathrm{~T}$. In fact, the magnetic field influence on the PL fine structure depends not only on the field magnitude, but also on some intrinsic properties and orientation of the quantum dot. In particular an increase of FSS is observed when the field is applied parallel to the orientation of the lower energy component of excitonic doublet $(\theta \approx 0$, see Fig. 22(a)), while a decrease is produced for perpendicular direction of the field $(\theta \approx \pi / 2$, see Fig $2(\mathrm{~b}))$.

Finally, we have also investigated some dots with a strongly tilted field configuration (i.e. $\theta \approx \pi / 4$ or $\theta \approx 3 \pi / 4)$. In such cases, in addition to the FSS

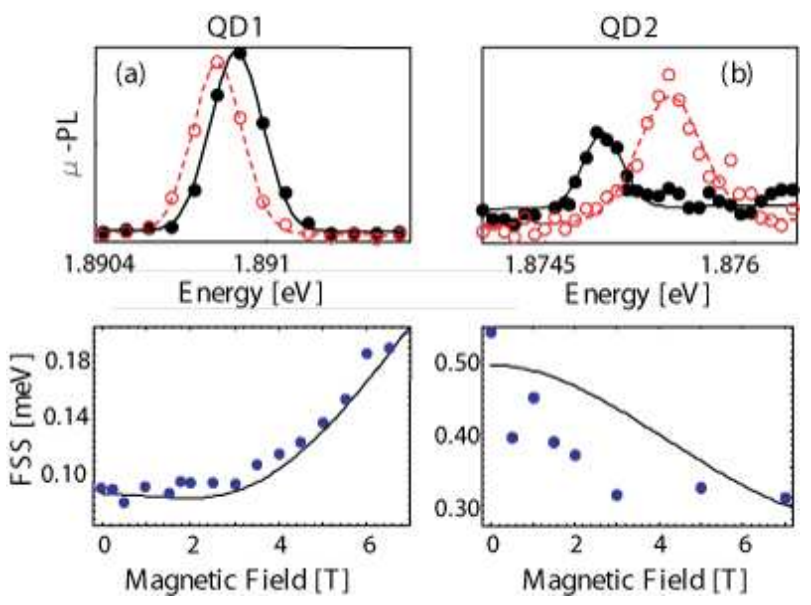

Figure 2: (Color online) Upper panel: $\mu$-PL spectra at $B=0 \mathrm{~T}$ for two dots ((a) and (b)) with perpendicular anisotropy orientations, $\theta=20^{\circ}$ and $\theta=110^{\circ}$, (open symbols and dashed line: measured at $20^{\circ}$, closed symbols and solid line: measured at $110^{\circ}$ ). Lower panel: Fine structure splitting (FSS) vs in-plane magnetic field $B$ for the dots from upper panel. Solid lines are theoretical curves according to the model discussed in the last section.
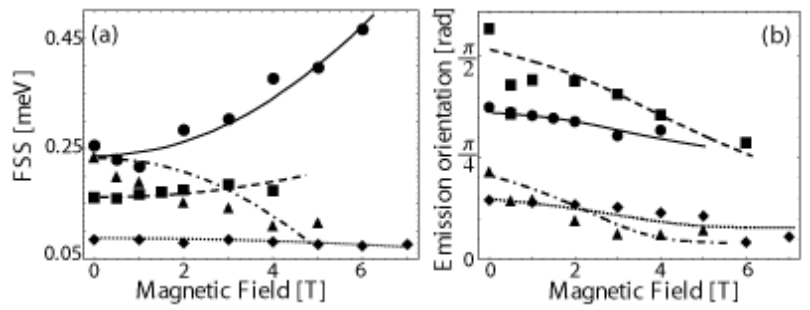

Figure 3: (a) Fine structure splitting (FSS), and (b) PL polarization orientation $\theta$ against in-plane magnetic field of four different QDs (different symbols). Lines are guide to the eyes.

change, the dot orientation shows a clear rotation when increasing the field. Figure 3 illustrates this effect for only a few selected dots, but observations were the same for all the QDs that we have studied (around 20). As shown, the reference QD eigenaxis rotates systematically towards the direction of the applied field. This indicates that the in-plane magnetic field $\boldsymbol{B}$ contributes to the FSS by an effective spin splitting of the bright exciton states characterized by the low (high) energy component polarized parallel (orthogonal) to the field. This conclusion is also supported by the corresponding FSS modification which depends both qualitatively and quantitatively on the initial angle $\theta$ between the field direction and QD orientation. The initial angle has to be close to $\pi / 2$ in order to get a reduction of FSS. If not, there is first a rotation of the optical orientation followed by an increase of FSS due to the field. The theoretical discussion presented below sheds some light on this qualitative description. 


\section{DISCUSSION}

The electron-hole exchange Hamiltonian responsible for the ground state exciton fine structure in an anisotropic quantum dot can be represented by 18 :

$$
\hat{H}_{e x}=\frac{\delta_{0}}{2} \hat{\sigma}_{z}^{e} \hat{\sigma}_{z}^{h}+\frac{\delta_{1}}{4}\left(\hat{\sigma}_{x}^{e} \hat{\sigma}_{x}^{h}-\hat{\sigma}_{y}^{e} \hat{\sigma}_{y}^{h}\right)+\frac{\delta_{2}}{4}\left(\hat{\sigma}_{x}^{e} \hat{\sigma}_{x}^{h}+\hat{\sigma}_{y}^{e} \hat{\sigma}_{y}^{h}\right)
$$

where the Pauli matrices $\sigma_{i}^{e, h}$ act on the spin components of the electron (e) or hole (h) respectively. Here, we used a $\pm 1 / 2$ pseudo-spin to describe the QD hole ground states with angular momentum $J_{z}=\mp 3 / 2$ along $z$. The quantities $\delta_{0}, \delta_{1}$, and $\delta_{2}$ describe the exciton quartet fine structure as follows : $\delta_{0}$ - between states of angular momentum $|M|=1$ and $|M|=2\left(\right.$ or $\sigma_{z}^{e}+\sigma_{z}^{h}=0$ ), $\left|\delta_{1}\right|$ (i.e. FSS) - between the components of the optically active doublet $(M= \pm 1)$, and $\left|\delta_{2}\right|$ - between the dark states $(M= \pm 2)$. These parameters are determined by the quantum dot properties (size, shape, composition, strain field, etc). In this formalism, the arbitrary $x, y$ directions of the Pauli matrices correspond to the eigenaxes of the QD. In the following we assume that the parameters $\delta_{0}, \delta_{1}$, and $\delta_{2}$ are not directly modified by the in-plane magnetic field, although for high field values the magnetic confinement likely affects the electron-hole exchange. Therefore, to the first order we only consider the Zeeman Hamiltonian to describe the effect of the inplane field $\boldsymbol{B}_{\perp}$ as recently done for self-assembled InAs QDs 10 . To derive properly the $g$ factor for hole ground states, we start from the general expression available for bulk excitons and given by $\underline{19}$ :

$$
\hat{H}_{Z}^{\text {bulk }}(\boldsymbol{B})=\mu_{B} \sum_{i=x, y, z}\left(\frac{1}{2} g^{e} \hat{\sigma}_{i}^{e} B_{i}-2 \kappa \hat{J}_{i} B_{i}-2 q \hat{J}_{i}^{3} B_{i}\right)
$$

where $\mu_{B}$ is the Bohr magneton, $g^{e}$ is the electron Landé factor, $q, \kappa$ are Luttinger coefficients and the $\hat{J}_{i}$ 's are the angular momentum projections of the Bloch states in the $\Gamma_{8}$ hole band along the crystallographic axes $\langle 100\rangle$. Usually, the main term driving the hole Zeeman splitting is the linear term $-2 \mu_{B} \kappa \hat{\boldsymbol{J}} \cdot \boldsymbol{B}$ while the cubic term in Eq.2 is considered as negligible. Yet, for a transverse magnetic field, only the hole states which differ by $|\Delta J|=1$ are coupled by $\hat{J}_{x}$ or $\hat{J}_{y}$. As a result, in the quantum dots investigated here, the hole ground states which are essentially pure heavy-holes with $J_{z}= \pm 3 / 2$ are not directly split by this term ${ }^{20}$. To obtain a non-zero transverse $g$ factor, which is required to modify the exciton $\mathrm{FSS}^{10}$, it seems thus necessary either to take into account the cubic term in Eq. 2] or to include in the model a light-hole doublet state $\left(J_{z}= \pm 1 / 2\right)$ split by an energy $\Delta_{h-l}$ of a few tens meV's from the hole ground state doublet. Actually, the sole Zeeman coupling to the light-hole states produces only a weak third-order contribution to the heavy hole splitting in a transverse magnetic field. We could conclude that only the cubic term contributes to the effective $g$ factor. However, including the light-holes also enables us to take into account the QD symmetry reduction to
$C_{2 v}$ or even $C_{2}$ (responsible for the FSS) which implies a direct coupling between the heavy and light hole ground states. The latter is proportional to the symmetrized product of the in-plane angular momentum components $\left\{\hat{J}_{x^{\prime}} \hat{J}_{y^{\prime}}\right\}^{18.21}$. Here, the indexes $x^{\prime}, y^{\prime}$ denote axes which are rotated by $\pi / 4$ with respect to the $\mathrm{QD}$ eigenaxes. After a $-\pi / 4$ rotation to use the same referential axes as Eq. (1I), we obtain the following Hamiltonian for the heavy-hole to light-hole coupling:

$$
\hat{H}_{h-l}=\beta\left(\hat{J}_{x}^{2}-\hat{J}_{y}^{2}\right)
$$

where $\beta$ represents the strength of the coupling. In the above formalism, based essentially on symmetry considerations, the respective signs of $\delta_{1}$ and $\beta$ are not a priori correlated although they are necessarily determined by the features of a given QD. This unknown sign correlation could however reveal of importance for the control of the FSS as discussed below ${ }^{22}$ and somehow enlightens the concept of "inverted"FSS in Ref. 10. Experimentally the perturbation $\hat{H}_{h-l}$ leads also to dichroism of the ground state excitonic transition (i.e. a difference in oscillator strength of the linearly-polarized doublet components) as reported in the past for the quantum wells of $C_{2 v}$ symmetry ${ }^{21}$ and more recently for trions in CdSe $\mathrm{QDs}^{23}$. It is worth mentioning that in InAs QD's similar dichroism has been reported and that no correlation was found between the sign of the linear polarization degree (related to $\beta$ ) and the sign of $\delta_{1}{ }^{24}$. For taking into account both $\hat{H}_{Z}^{\text {bulk }}$ and $\hat{H}_{h-l}$ we have to introduce the angle $\phi$ between the QD eigenaxis $x$ and the crystallographic direction [100] in order to rotate the cubic term of $\hat{H}_{Z}^{\text {bulk }}$ in the QD coordinate frame (see Fig. (4). In this way, we obtain the effective Zeeman Hamiltonian to the first order in $\beta / \Delta_{h-l}$ and in the basis of electron spin and hole pseudo-spin :

$$
\hat{H}_{Z}\left(\boldsymbol{B}_{\perp}\right)=\frac{1}{2} \mu_{B}\left(\sum_{i} g^{e} \hat{\sigma}_{i}^{e} B_{i}+\sum_{i, j} \hat{\sigma}_{i}^{h} g_{i j}^{h} B_{j}\right)
$$

with the hole $g$ factor tensor :

$$
\begin{aligned}
g^{h} & =3 q\left(\begin{array}{cc}
\cos 4 \phi+\rho_{g} & \sin 4 \phi \\
-\sin 4 \phi & \cos 4 \phi-\rho_{g}
\end{array}\right) \\
\text { where } \rho_{g} & =\frac{(4 \kappa+7 q) \beta}{q \Delta_{h-l}}
\end{aligned}
$$

As it could be expected, the transverse hole $g$ factor gets anisotropic due to the term proportional to $\beta$ as already emphasized in Ref. 23. Of course, the parameters $g^{e}, \kappa$ and $q$ in Eqs. 45 are not the bulk parameters of a quantum dot (or a barrier) material: in QDs the strong confinement of eigenstates considerably affects the values of these parameters as predicted ${ }^{25.26}$ and experimentally observed 17 . In particular the mixing allowed in $D_{2 d}$ symmetry between heavy-hole and light-hole with envelope wave functions of different angular momentum 
may explain the significative value often reported for the transverse hole $g$ factor ${ }^{20}$. In the following we assume first a symmetric transverse $g$ factor for the hole $(\beta=0)$ and then discuss the effect produced by an antisymmetric term $(\beta \neq 0)$.

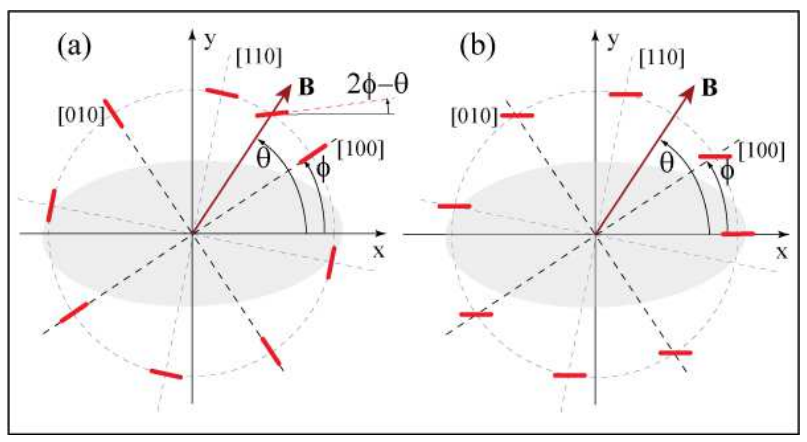

Figure 4: (Color online) Schematics of the crystallographic axis configuration with respect to the QD principal axes $x, y$. Thick red dashes represent the exciton polarization orientation produced by the field-induced splitting only for different directions of the field. (a) Effect of the cubic term (see text), (b) effect of the linear term, including heavy-hole to light-hole coupling. The grey shaded area represents a QD elongated along the polarization eigenaxis $x$.

The total Hamiltonian $\hat{H}_{e x}+\hat{H}_{Z}$ enables us to predict the evolution of the bright state FSS as a function of $\boldsymbol{B}_{\perp}$. The results are displayed in Fig. [5] which shows the absolute splitting of the bright excitons (panel (a)) and their polarization rotation angle $\Delta \theta$ (panel (b)). Both are plotted as a function of field magnitude and orientation represented here by $\theta-2 \phi$. Before commenting further these figures, let us consider the effect of the cubic term in $\hat{H}_{Z}$ for a symmetrical QD with $\delta_{1}=0$ (and $\beta=0)$. In this case, the choice of the $\mathrm{QD} x, y$ axes to define the angles $\theta$ and $\phi$ is arbitrary. Choosing $\phi=0$ shows that the hole $g$ factor tensor reduces to the scalar value $3 q$ which leads to an isotropic FSS induced by the field when its orientation is varied. On the other hand, if we fix $\theta=0$ we observe that in the referential attached to the field, the polarization of the upper excitonic doublet split by the field rotates faster by an angle $2 \phi$ than the field from the [100] axis. This is illustrated in the left-hand part of Fig. 4. In the general case, it is thus clear that depending on the field direction $\theta$, the fieldinduced splitting will add to or subtract from an initial finite splitting $\delta_{1}$. Calculations presented in Fig. 5] (a) show that cancelation can be achieved for $\theta=\pi / 2+2 \phi$ (changing the sign of $q$ with respect to $g^{e}$ shifts this angle by $\pm \pi / 2$ ), i.e. for a field oriented symmetrically to the low energy component of the bright doublet ( $y$ axis) with respect to the crystallographic direction [100]. A small discrepancy of the field orientation leads to a continuous rotation of the eigenaxes directions when passing near the critical point $\left(B_{\text {crit. }}, \pi / 2+2 \phi\right)$ of exact cancelation as shown in Fig. 5(b). Nevertheless, choosing correctly the field direction with respect to the QD orientation allows in principle to reduce the FSS of any QDs. This point was not clearly established in the analytical treatment presented by Stevenson et $a l^{10}{ }^{10}$ where however only the isotropic contribution of Eq. (4) was considered 27.
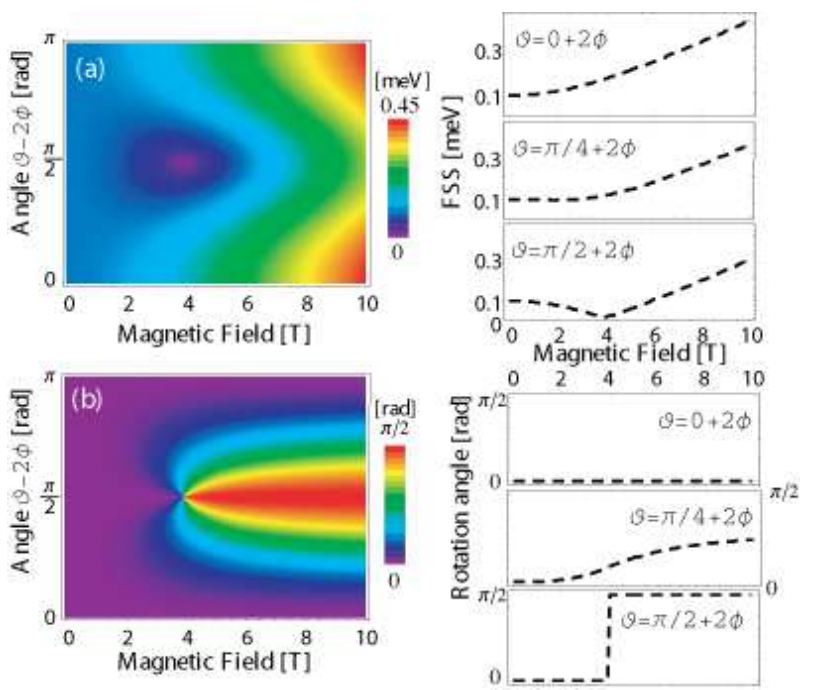

Figure 5: (a) FSS absolute value vs in-plane field direction $\theta-2 \phi$ and magnitude encoded on a color scale. Cross-sections for three directions of the field are displayed on the right-hand side. (b)Rotation angle $\Delta \theta$ of the PL polarization orientation vs in-plane magnetic field direction $\theta$ and magnitude. Three cross-sections for $\theta=0, \pi / 4$ and $\pi / 2$ are also shown. Calculations are made with an isotropic $g$ factor $(\beta=0), \delta_{0}=80 \mu \mathrm{eV}$ and a FSS $\delta_{1}=80 \mu \mathrm{eV}$ in zero field.

Taking now into account the $g$ factor anisotropy $\left(\rho_{g} \propto\right.$ $\beta \neq 0)$ may considerably change the above phenomenology. As shown in Fig. [6] the key feature turns out to be the sign of $\rho_{g}$ (determined by that of $\kappa \beta$ ) with respect to $\delta_{1}$. If opposite, we find that it is still possible to reduce to zero the FSS for $\theta=\pi / 2+2 \phi$, and actually when the antisymmetric part really dominates $\left(\left|\rho_{g}\right| \gg 1\right)$ this can be achieved for any field direction. On the contrary, for $\rho_{g}$ and $\delta_{1}$ of same sign the critical field $B_{\text {crit. }}$ for which cancelation could be achieved, diverges when $\left|\rho_{g}\right|$ approaches 1, a situation which indeed corresponds to $g_{y y}^{h}=0$. For larger values of the anisotropy the magnetic field produces an increase of the FSS whatever its in-plane orientation $\theta$ is, as illustrated in Fig. 6(b). In both cases, when the anisotropy dominates $\left(\left|\rho_{g}\right| \gg 1\right)$ the principal axes of the PL polarization remain essentially parallel to their initial orientation (see Fig. 4(b)), in contrast to the case $\beta=0$ as obvious in Fig. [5] for fields above $\sim 4 \mathrm{~T}$. Our analysis reveals thus that in the case of a strong anisotropy of the hole $g$ factor the possibility of tuning the QD FSS by a magnetic field depends essentially on the sign of $\beta$ with respect to $\delta_{1}$.

In our experiments we did not vary the in-plane field orientation $\theta$ for a given QD. Therefore it turns out 

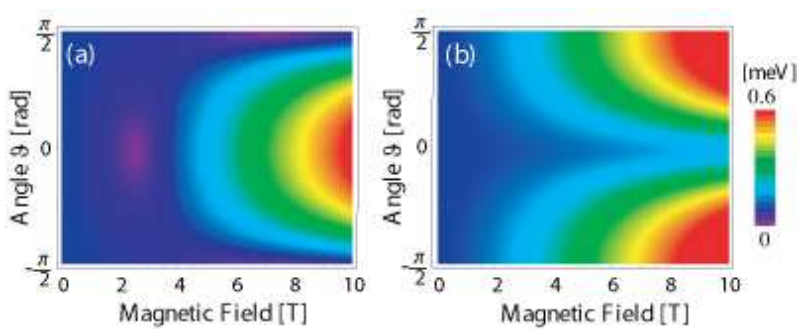

Figure 6: FSS absolute value on a color scale vs in-plane field direction $\theta-2 \phi$ and magnitude for a strong $g$ factor anisotropy $\rho_{g}$. Depending on its sign with respect to the initial splitting (here $\delta_{1}=80 \mu \mathrm{eV}$ ) it produces very different behaviors : (a) $\rho_{g}=-1.3$, (b) $\rho_{g}=+1.3$. We used the same average $g$ factor $\bar{g}_{h}=0.78, g^{e}=1.75$ and $\delta_{0}=0.8 \mathrm{meV}$ as in Fig. [5]

to be rather delicate to determine the strength of the in-plane $g$ factor anisotropy. Yet, the fact that in most cases we observed a rotation of the QD principal axes towards those defined by the field indicates that the average value $(3 q)$ of the $g$ factor likely dominates over its anisotropic contribution $\propto \beta$. Besides, we also did not observe significative degree of linear polarization between the PL intensities of both exciton components, which means that $\beta / \Delta_{h-l} \ll 1$. This situation clearly differs from that reported for CdSe quantum $\operatorname{dots}^{23}$.

For the quantum dot QD1 shown in Figs. 102 we could produce a good fit of the FSS evolution by taking the values of $\delta_{1}\left(\delta_{0}\right)$ observed (extrapolated) in zero magnetic field, and $\delta_{2}$ assumed to be around $1 \mu \mathrm{eV}$. The only fitting parameters were thus the electron and hole $g$ factors considered as isotropic. We included in the model the field orientation $\theta$ with respect to the dot main axis, which also defines $\phi$, as in our experiment the field was parallel to one of the cleaved edge of the sample corresponding to $|\theta-\phi|=\pi / 4$. We obtained the following $g$ factor values $\left|g^{e}\right|=1.75 \pm 0.1$ and $\left|g^{h}\right|=0.78 \pm 0.1$ for the fit shown in Fig. 1 The decrease of FSS observed for QD2 (characterized by $\theta \approx \pi / 2$ ) could also be reproduced by the model with values $\left|g^{e}\right|=1.75 \pm 0.2$ and $\left|g^{h}\right|=0.9 \pm 0.2$. The dark states being absent from the spectra, $\delta_{0}=1 \mathrm{meV}$ was arbitrary chosen for the latter fitting. In the general case of tilted QD orientation the almost systematic rotation of the PL polarization towards the field direction $(\theta+\Delta \theta \rightarrow 0$ in Fig. 3(b)) agrees well with our model with a negligible $g$ factor anisotropy. But clearly, further experimental investigations consisting in a full mapping of the influence of the magnetic field on FSS as a function of its in-plane orientation and magnitude should be performed to determine more quantitatively the $g$ factor anisotropy in these quantum dots.

In all studied QDs, the observed dark states were fully linearly polarized (see for example Fig. 1(a)) and we noticed only one component of dark excitonic doublet. The lack of one "dark" state in the spectrum can be explained within our interpretation. For the used experimental con- figuration $\phi=\pi / 4$, and for quantum dots with eigenaxes parallel to the crystallographic direction [110] or [-110] $(\theta=0$ or $=\pi / 2)$ we obtain the Hamiltonian in a particularly simple form. It may be used to describe the case of QD1, for which $\theta$ is close to zero. After writing down the discussed Hamiltonian in a basis of linearly polarized states $^{28}$ it has a following form:

$$
\begin{aligned}
& \hat{H}_{Q D 1}= \\
& \frac{1}{2}\left(\begin{array}{cccc}
\delta_{0}+\delta_{1} & 0 & \mu_{B} B\left(g^{e}+g^{h}\right) & 0 \\
0 & \delta_{0}-\delta_{1} & 0 & \mu_{B} B\left(g^{e}-g^{h}\right) \\
\mu_{B} B\left(g^{e}+g^{h}\right) & 0 & -\delta_{0}+\delta_{2} & 0 \\
0 & \mu_{B} B\left(g^{e}-g^{h}\right) & 0 & -\delta_{0}-\delta_{2}
\end{array}\right)
\end{aligned}
$$

The diagonal elements refer to energy levels of states in absence of magnetic field, the non-diagonal ones show the mixing induced by the field. In this representation it is clearly visible that mixing of dark and bright states occurs independently for each linear polarization. The mixing matrix elements for $x$ and $y$ polarizations are proportional either to $\left(g^{e}+g^{h}\right)$ or to $\left(g^{e}-g^{h}\right)$, respectively. The corresponding intensities of the transitions are proportional to the squares of corresponding matrix elements. For the obtained values of g-factors the theoretically predicted intensity ratio for dark transitions is 7.84. It explains qualitatively the strong asymmetry in the intensities of the two components of the dark excitonic doublet.

\section{CONCLUSION}

In summary, we have shown that an in-plane magnetic field modifies the fine structure splitting of the excitonic emission of $\mathrm{CdTe} / \mathrm{ZnTe}$ quantum dots. This effect depends on the field direction. If applied along one of the main axes of the dot, the field can either increase or decrease the splitting. If not, a rotation of the bright exciton eigenaxes towards the axis defined by the field direction is generally observed together with a change of the splitting. These effects are in qualitative (polarization rotation) and rough quantitative (splitting variation) agreement with a simple model based on a Zeeman spin Hamiltonian. We find that in the QDs investigated here the anisotropy of the $g$ factor is likely negligible, in contrast to results reported for other types of self-assembled QDs. This could be an advantage as in this case the possibility to cancel the fine structure splitting does not seem to be hindered by light-heavy hole mixing.

\section{Acknowledgments}

This work has been partially supported by Polish Ministry of Science and Higher Education (Grants 1PO3B-114-30 and 2PO3B-015-25). One of us (K.K.) 
is supported by the European network of excellence SANDIE. We would like to thank Pr. E. Ivchenko for enlightening discussions.
* Corresponding author kowalik@lpn.cnrs.fr

1 N. Akopian, N. H. Lindner, E. Poem, Y. Berlatzky, J. Avron, D. Gershoni, B. D. Gerardot, and P. M. Petroff, Phys. Rev. Lett. 96, 130501 (2006).

2 R. M. Stevenson, R. J. Young, P. Atkinson, K. Cooper, D. A. Ritchie, and A. J. Shields, Nature 439, 179 (2006).

3 R. J. Young, R. M. Stevenson, P. Atkinson, K. Cooper, D. A. Ritchie, and A. J. Shields, New J. Phys. 8, 29 (2006).

${ }^{4}$ G.Bester and A. Zunger, Phys. Rev. B 71, 045318 (2005).

5 R. Seguin, A. Schliwa, S. Rodt, K. Pötschke, U. W. Pohl, and D. Bimberg, Phys. Rev. Lett. 95, 257402 (2005).

${ }^{6}$ R. J. Young, R. M. Stevenson, A. J. Shields, P. Atkinson, K. Cooper, D. A. Ritchie, K. M. Groom, A. I. Tartakovskii, and M. S. Skolnick, Phys. Rev. B 72, 113305 (2005).

7 A. I. Tartakovskii, M. N. Makhonin, I. R. Sellers, J. Cahill, A. D. Andreev, D. M. Whittaker, J.-P. R. Wells, A. M. Fox, D. J. Mowbray, M. S. Skolnick, et al., Phys. Rev. B 70, 193303 (2004).

${ }^{8}$ K. Kowalik, O. Krebs, A. Lemaître, S. Laurent, P. Senellart, J. A. Gaj, and P. Voisin, Appl. Phys. Lett. 86, 041907 (2005).

9 S. Seidl, M. Kroner, A. Högele, K. Karrai, R. Warburton, A. Badolato, and P. M. Petroff, Appl. Phys. Lett. 88, 203113 (2006).

10 R. M. Stevenson, R. J. Young, P. See, D. G. Gevaux, K. Cooper, P. Atkinson, I. Farrer, D. A. Ritchie, and A. J. Shields, Phys. Rev. B 73, 033306 (2006).

11 F. Tinjod, S. Moehl, K. Kheng, B. Gilles, and H. Mariette, J. Appl. Phys. 95, 102 (2004).

12 J. Puls, M. Rabe, H.-J. Wünsche, and F. Henneberger, Phys. Rev. B 60, 16303(R) (1999).

13 F. Tinjod, B. Gilles, S. Moehl, K. Kheng, and H. Mariette, Appl. Phys. Lett. 82, 4340 (2003).

14 J. Jasny, J. Sepiol, T. Irngartinger, M. Traber, A. Renn, and U. P. Wild, Rev. Sci. Instrum. 67, 1425 (1996).

15 A. Kudelski, A. Golnik, J. A. Gaj, S. Mac̀kowski, G. Karczewski, and J. Kossut, in PROC. 25TH Int. Conf. Phys. Semicond. (Springer, Osaka, 2000), p. 1249.

${ }^{16}$ L. Marsal, L. Besombes, F. Tinjod, K. Kheng, A. Wasiela, B. Gilles, J.-L. Rouvière, and H. Mariette, J. Appl. Phys. 91, 4936 (2002).
17 M. Bayer, G. Ortner, O. Stern, A. Kuther, A. A. Gorbunov, A. Forchel, P. Hawrylak, S. Fafard, K. Hinzer, T. L. Reinecke, et al., Phys. Rev. B 65, 195315 (2002).

18 E. I. Ivchenko and G. E. Pikus, Superlattices and Other Heterostructures, Symmetry and Optical Phenomena (Springer-Verlag, Berlin, 1995).

19 H. van Kesteren, E. Cosman, W. van der Poel, and C. T. Foxon, Phys. Rev. B 41, 5283 (1990).

${ }^{20}$ For a quantum dot with $D_{2 d}$ symmetry, the projection of the total angular momentum $\hat{M}_{z}=\hat{L}_{z}^{\text {envelope }}+\hat{J}_{z}$ is a good quantum number and the hole ground states correspond to $M_{z}= \pm 3 / 2$. Yet, due to the coupling of heavy-hole to light-hole enabled at $\boldsymbol{k}_{\perp} \neq 0$ by a term $\propto\left(k_{x}^{2}-k_{y}^{2}\right)$ in the $\Gamma_{8}$ Luttinger Hamiltonian, they are not eigenstate of $\hat{J}_{z}$.

21 A. A. Toropov, E. L. Ivchenko, O. Krebs, S. Cortez, P. Voisin, and J. L. Gentner, Phys. Rev. B 63, 35302 (2000).

22 The coupling described by Eq. 3 contributes to $\delta_{1}$ through the short-range electron-hole exchange $-\frac{4}{3} \delta_{0} \hat{\boldsymbol{\sigma}}^{e} \cdot \hat{\boldsymbol{J}}$, by an amount equal to $2 \beta \delta_{0} / \Delta_{h-l}^{2}$. However, the dominant contribution in $\delta_{1}$ is supposed to originate from the long-range part of the electron-hole exchange which as a result does not permit to establish the sign correlation with $\beta$.

23 A. V. Koudinov, I. A. Akimov, Yu. G. Kusrayev, and F. Henneberger, Phys. Rev. B 70, 241305(R) (2004).

24 I. Favero, G. Cassabois, A. Jankovic, R. Ferreira, D. Darson, C. Voisin, C. Delalande, P. Roussignol, A. Badolato, P. M. Petroff, et al., Appl. Phys. Lett. 86, 041904 (2005).

25 A. A. Kiselev, E. L. Ivchenko, and U. Rössler, Phys. Rev. B 58, 16353 (1998).

26 C. E. Pryor and M. E. Flatté, Phys. Rev. Lett. 96, 026804 (2006).

27 For InAs QDs, the polarization eigenaxes are in most cases parallel to the $\langle 110\rangle$ crystallographic axes leading to $\phi=$ $\pi / 4$. As a result $g^{h}$ gets diagonal and actually is equal to the scalar factor $3 q$ when no heavy-hole light-hole coupling is included.

28 Normalized sum ( $x$-polarized) and difference ( $y$-polarized) of bright $M= \pm 1$ states followed by sum and difference of $M= \pm 2$ dark states. 